Cahiers d'études italiennes

\title{
L'ottica infantile sulla guerra e sulla violenza
}

\section{Claudia Nocentini}

\section{OpenEdition}

\section{Journals}

Edizione digitale

URL: http://journals.openedition.org/cei/270

DOI: $10.4000 /$ cei. 270

ISSN: 2260-779X

Editore

UGA Éditions/Université Grenoble Alpes

Edizione cartacea

Data di pubblicazione: 15 juin 2005

Paginazione: 23-28

ISBN: 978-2-84310-066-6

ISSN: $1770-9571$

Notizia bibliografica digitale

Claudia Nocentini, «L'ottica infantile sulla guerra e sulla violenza», Cahiers d'études italiennes [Online], 3 2005, online dal 15 décembre 2006, consultato il 27 mars 2021. URL: http://journals.openedition.org/ cei/270 ; DOI: https://doi.org/10.4000/cei.270 


\title{
L'OTTICA INFANTILE \\ SULLA GUERRA E SULLA VIOLENZA
}

\author{
Claudia Nocentini \\ University of Edinburgh
}

In questo studio mi propongo di analizzare la rappresentazione dell'infanzia e/o prima adolescenza in Il sentiero dei nidi di ragno e Il cielo è rosso, due romanzi scritti a ridosso della seconda guerra mondiale ${ }^{1}$. In un recente video sull'opera di Calvino, Ceserani ha commentato che la centralità della figura infantile nella poetica neorealista è particolarmente evidente nella produzione cinematografica ${ }^{2}$. Ma anche a prescindere dalla poetica neorealista mi sembra che l'inserzione di un personaggio infantile in un racconto di guerra o di dopoguerra - cinematografico, narrativo o teatrale - faccia appello al senso di responsabilità dell'adulto, risvegliando l'istinto protettivo dello spettatore o del lettore. Così in Napoli milionaria di Eduardo De Filippo del 1945, in scena ancora prima della fine della guerra, il fragile destino nazionale è trasposto in quello della piccola Rita, la bambina malata a proposito della quale il padre pronuncia la famosa battuta " Ha da passa" 'a nuttata ${ }^{3}$ ". Nello stesso modo il binomio genitore-figlio rappresenta il tentativo di assicurare la sopravvivenza dei più deboli, o anzi della parte più debole di noi, come dimostra in particolare il rapporto padre-figlio in opere che vanno da Ladri di biciclette a La vita ̀̀ bella, e senza dubbio anche oltre. Ma basta contrapporre il comporta-

1. Il sentiero dei nidi di ragno fu pubblicato da Einaudi nel 1947 (cito dall'edizione critica delle opere di Calvino, Romanzi e Racconti, Milano, Mondadori, 1991). La perduta gente fu pubblicato da Longanesi nel 1947 col titolo Il cielo è rosso. Cito dall'edizione Rizzoli, 2001.

2. Calvino (2 videocassette) a cura di D. Brogi, Palumbo Editore, 2000. Sulla raffigurazione del bambino nel cinema italiano del dopoguerra si veda anche G. Grazzini, Dolci, pestiferi e perversi, Pratiche Editrice, 1995.

3. Eduardo De Filippo, Napoli milionaria in Cantata dei giorni dispari, Torino, Einaudi, 1957, p. 127. 
mento dei padri in questi due film per vedere la necessità di ulteriori distinzioni : da una parte il cedimento alla tentazione, tanto condannabile quanto comprensibile, di " adattarsi alle circostanze " nel film di De Sica, e dall'altra l'eroismo dissimulato attraverso la comicità del film di Benigni, capace, data la distanza temporale, di raffigurare il destino tragico di una famiglia italiana perfetta in tutto e per tutto.

Nei testi che ho scelto di studiare, la figura infantile o adolescenziale è ancora più centrale perché i protagonisti in questione sono addirittura orfani. In quanto testi di finzione, corrispondono alla precisa volontà autoriale di impostare il racconto da un punto di vista di estrema vulnerabilità, quello della vittima innocente, o, dal punto vista dello stato civile, di minori senza tutela. Prima di passare alla lettura dei testi, sarà il caso di introdurre brevemente i loro autori e le circostanze in cui sono stati scritti.

Italo Calvino, nato a Santiago di Avana, a Cuba nel 1923 da genitori scienziati, laici e antifascisti, aveva passato buona parte dell'infanzia e dell'adolescenza a Sanremo, ma nei primi anni di guerra si era trovato a Torino e a Firenze come studente universitario. Interrotti gli studi dopo la caduta di Mussolini nel '43, era tornato a Sanremo e aveva preso parte alla Resistenza fra i partigiani delle brigate Garibaldi. Tornato a Torino nel primo dopoguerra, aveva cominciato a scrivere racconti e piccoli saggi per giornali e periodici progressisti come L'Unità e Il Politecnico ed era entrato in rapporti di amicizia con il team di intellettuali della casa editrice Einaudi. In particolare Cesare Pavese e Natalia Ginzburg, suoi primi lettori, verso la fine del 1946 lo avevano incoraggiato a scrivere un racconto di respiro più lungo e a concorrere ad un premio letterario presso la casa editrice Mondadori : così $\mathrm{Il}$ sentiero dei nidi di ragno fu scritto nel dicembre del 1946 e pubblicato da Einaudi nel $1947^{4}$.

Giuseppe Berto, nato a Mogliano Veneto nel 1914, figlio di un maresciallo dell'esercito, aveva frequentato il liceo in un collegio salesiano a Treviso e si era laureato in lettere all'università di Padova nel 1940. Volontario in Africa orientale nel 1939, a causa di una invalidità di guerra fu scartato dall'esercito regolare, ma riuscì a tornare in Libia nel 1942, ancora una volta come volontario, nel IV Battaglione camicie nere. Catturato dalle forze alleate nel maggio del 1943, scrisse nel campo di prigionia di Hereford nel Texas il lungo racconto La perduta gente, pubblicato da Longanesi nel 1947 col titolo Il cielo è rosso che risultò svincitore del premio Firenze del 1949. Nel 1955 pubblicò (presso Garzanti) un

4. Si veda Album Calvino a cura di L. Baranelli e E. Ferrero, Palomar SRL e Mondadori, 1995. 
racconto-diario della seconda esperienza in Africa, intitolato Guerra in camicia nera e nel 1964 il suo libro più famoso, Il male oscuro, premio Viareggio e Campiello, il diario della propria esperienza psicoanalitica condotta con un analista freudiano della scuola di Weiss 5.

Fra i due testi ci sono molte differenze di cui non parlerò ma che val la pena di elencare : di lunghezza (142 pagine di Calvino contro 400 di Berto), di qualità (tanto pare nitido ed essenziale il testo di Calvino, quanto si protrae fra molte incertezze quello di Berto), di presentazione (filtro cinico-espressionista per Calvino e psicologico-sentimentale quello di Berto), di vicinanza alla realtà storica (si può obiettare che la tematica dibattuta nel decimo capitolo di Calvino riflette meglio il clima del dopoguerra che quello del conflitto, ma questo è descritto realisticamente, mentre il testo di Berto ritrae una guerra immaginaria, nella quale i tedeschi non vengono nemmeno nominati e gli unici stranieri in Italia sembrano essere gli americani). Quello che questi due testi hanno in comune è la scelta di rappresentare il conflitto attraverso gli occhi di minori indifesi.

Nel testo di Calvino il piccolo Pin e sua sorella sono orfani di madre; il padre è ancora vivo, ma li ha abbandonati. In quello di Berto, dei cinque giovanissimi protagonisti, all'inizio del racconto solo Giulia, figlia di una prostituta, è senza padre e alla fine solo Carla lo ha ancora, ma compare non come figura protettiva, bensì come personaggio dipendente e manipolativo che reclama un sostegno economico.

Tutti e due i testi sono in terza persona. Calvino limita il racconto al punto di vista di Pin (con l'eccezione di un capitolo in cui la focalizzazione è affidata a un giovane commissario partigiano), e circoscrive la narrazione al periodo dell'occupazione tedesca prima della fine della guerra. Berto comincia il racconto nel gennaio del 1931 e lo conclude in un dopoguerra indeterminato. Usa una tecnica multifocale, spostandosi progressivamente dalla madre a Giulia, e da questa a Daniele. La vicenda si può riassumere così : la madre di Giulia muore, affidandola bambina alla nonna e allo zio. Nel 1940 sono rimaste sole con la nonna tanto Giulia di nove anni quanto sua cugina Carla di dieci anni, il cui padre è in prigione e la cui madre è andata a cercare lavoro a Napoli. A quattordici anni Carla e il diciassettenne Tullio instaurano un rapporto sessualmente attivo. Nel

5. Si vedano E. Ghidetti, e G. Luti, Dizionario critico della Letteratura italiana del Novecento, Roma, Editori Riuniti, 1997, p. 71-73 ; Giuseppe Berto vent'anni dopo, Atti del Convegno, Padova-Mogliano Veneto, 23-24 ottobre 1998, a cura di S Bartolomeo e S. Chemotti, introduzione di C. De Michelis, Pisa-Roma, Istituti Editoriali e Poligrafici Internazionali ; D. Biagi, Vita scandalosa di Giuseppe Berto, Torino, Bollati Boringhieri, 1999. 
1944, durante un violento bombardamento muoiono la nonna delle ragazze e i genitori di Tullio ${ }^{6}$. Pur senza casa e famiglia Giulia, Carla e Tullio si fanno carico di un gruppo di piccoli orfani e in particolare di Maria, una piccola ritardata. Alloggiano abusivamente in un quartiere cittadino evacuato perché pericolante. Tullio organizza furti per sopravvivere e a scopo caritatevole, accogliendo in casa Daniele, liceale orfano in fuga da un collegio di preti. Carla, il cui rapporto con Tullio si è deteriorato fin dal bombardamento, è diventata una prostituta e s'innamora di Daniele. Quest'ultimo non ricambia il suo amore per lealtà nei confronti di Tullio che ammira sia per la sua intraprendenza pratica sia per la sicurezza che gli danno le sue convinzioni politiche comuniste. Nel primo dopoguerra Tullio viene ucciso mentre assale un convoglio militare. Daniele trova un paio di piccoli lavori e s'innamora, ricambiato, di Giulia con cui divide la maggior parte del suo tempo nell'interno pericolante in cui vivono. Caratterizzata come un personaggio di particolare sensibilità fin dall'infanzia, Giulia è malata e muore di tubercolosi. Daniele, incapace di restare dopo la sua morte, lascia Carla e Maria al loro destino, si allontana e si suicida.

Nonostante la grande differenza fra il registro cinico-espressionista di Calvino e quello sentimentale e decadente di Berto, tutti e due i testi rappresentano dei bambini molto più responsabili degli adulti. Se si eccettuano Kim e Ferriera, i due interlocutori positivi del capitolo decimo che per altro contribuiscono all'azione unicamente in funzione di commento storico, solo il marinaio tedesco Frick, il commissario Giacinto e il cuoco Mancino, descritti come vittime delle circostanze, sono leggermente antieroici. Tutti gli altri lo sono marcatamente, e incarnano una galleria di vizi - dalla lussuria alla codardia, dalla pigrizia all'ira - le cui conseguenze sono la distruzione delle comunità, cittadina prima e partigiana poi, di cui fanno parte.

Altrettanto grave il degrado rappresentato da Berto, in un mondo adulto caratterizzato dall'interesse economico. La decisione di una prostituta di portare a termine una gravidanza contro il parere di sua madre e di suo fratello (che pure, ovviamente, essa mantiene), un'elemosina di un signore per strada e il dono di un paio di guanti da parte di un soldato americano sono gli unici atti adulti che non siano dettati dal tornaconto. Questo vale tanto per il contesto sottoproletario di Carla e Giulia quanto per quello borghese di Daniele il quale, dopo la morte dei genitori, non

6. La descrizione del bombardamento e il guastarsi del rapporto fra i giovani amanti ricalca l'esperienza dell'autore a Tunisi in Guerra in camicia nera, Milano, Garzanti, 1955, p. 178-9. 
mostra per lui nessuna forma di solidarietà. Né il collegio religioso, né il nonno distante, né gli amici di famiglia mostrano comprensione o affetto per l'adolescente orfano.

La mancanza di generosità del mondo adulto ha caratteristiche lievemente diverse nei due libri. Nell'atmosfera combattente del testo di Calvino significa mancanza di valore, in quello di Berto di valori che permettano la sopravvivenza esistenziale. In questa condanna del mondo adulto mi pare sia da leggere una condanna della società italiana sotto il fascismo. Tanto la famiglia di Pin quanto quella di Carla e Giulia sono economicamente povere, ma soprattutto sono famiglie moralmente povere e non c'è istituzione pubblica o religiosa in grado di provvedere alle loro lacune. Nel testo di Calvino la denuncia è implicita nell'avversione di Pin per fascisti e preti e nella sua scarsa alfabetizzazione. Paradossalmente, date le sue opinioni politiche ${ }^{7}$, questa condanna è tanto più marcata ed esplicita nel testo di Berto, che ritrae prima un insegnante-sacerdote dalla scarsa sensibilità e dall'alto livello di fanatismo, e poi un vecchio maestro fascista aiutato dai ragazzi nonostante la retorica vuota delle sue parole. Nell'atmosfera autocritica del primo dopoguerra il punto di vista infantile o giovanile è l'unico senza colpa. (Ma anche i bambini non sono senza difetti, in particolare Pin, talmente volgare e maleducato che ha bisogno di una voce narrante adulta in grado di spiegare al lettore le ragioni di tanto degrado ${ }^{8}$ ).

In contrasto con l'egoismo irresponsabile degli adulti di Calvino e la meschinità di quelli di Berto, brilla il merito dei piccoli. Ne Il sentiero dei nidi di ragno il furto della pistola tedesca e il rifiuto di cedere alle pressioni della milizia da parte di Pin, l'evasione dalla prigione fascista e l'uccisione di Pelle da parte di Lupo Rosso, sono rappresentati come atti di coraggio. Benché non siano lodati in modo altrettanto esplicito, sono coraggiosi anche i furti redistributivi alla Robin Hood del comunista Tullio e la prostituzione di Carla, i due personaggi forti de Il cielo è rosso, mentre la mag-

7. Le opinioni politiche di Berto durante la guerra potrebbero essere riassunte dalla seguente citazione: "Due sono i fattori che mi hanno spinto a cercare, con una certa insistenza, di venire in guerra, io che mi sento responsabile nella misura giusta, cioè quanto ne spetta a ciascun italiano che abbia capacità di intendere e di volere. Se non si volevano il fascismo e la guerra, bisognava pensarci prima. Ora ne siamo tutti più o meno responsabili e starsene inerti a guardare gli avvenimenti è la cosa più vile che si possa fare. Da quando è scoppiata la guerra e fin che durerà, l'identificazione del fascismo con l'Italia non è da discutersi. Il secondo fattore nasce dalla considerazione che in Italia le cose non vanno affatto bene e che, dopo la vittoria, bisognerà porci un rimedio, a costo di fare un'altra rivoluzione. L'aver partecipato con onore a questa guerra costituirà un buon diritto per fare la rivoluzione, e io voglio trovarmi tra coloro che la faranno " In G. Berto, Guerra in camicia nera, op. cit, p. 24.

8. Si veda C. Nocentini Narrative and Ideological Function of the Narrator in Il sentiero dei nidi di ragno in "Forum for Modern Language Studies ", vol. XXXIII, n.4, 1997, p. 369-375. 
giore sensibilità di Giulia e Daniele è chiaramente in rapporto diretto con il loro rifiuto di adattarsi alle circostanze e di sopravvivere.

La prostituzione pare essere l'unica attività redditizia per le donne in generale. Nel testo di Calvino, Nera, la sorella di Pin, giovane ma di età indeterminata, fa la prostituta, e nel testo di Berto sono prostitute tanto la madre di Giulia, morta prima del 1940, quanto sua cugina Carla. Data l'invisibilità e la marginalità del lavoro femminile durante il fascismo e le mancanze di impiego durante il conflitto, la scelta di rappresentarle in questa attività pare ragionevolmente realistica. E non stupisce che l'unica clientela riconoscibile delle giovani prostitute durante il conflitto sia di provenienza straniera e/o nemica : il tedesco Frick e i militi della brigata nera nel testo di Calvino, gli americani in quello di Berto. L'atteggiamento autoriale nei confronti della prostituzione si direbbe di netta condanna nel Sentiero, in cui Nera viene liquidata dal misogino partigiano Cugino, e di pietosa condiscendenza ne Il cielo è rosso, in cui Carla, per quanto abbandonata con gravi pesi, è lasciata sopravvivere. Ma non c'è dubbio che il testo condanna assai più la prostituzione di Carla che non il furto di Tullio. Il fatto che alla fine del racconto Carla sia appena quindicenne e ogni suo cliente sarebbe perseguibile penalmente forse non ha bisogno di menzione, ma di certo non viene mai menzionato, mentre la sua caratterizzazione indugia sulla spavalderia con cui affronta quello che gli altri personaggi ritengono piuttosto apertamente una colpa ?

Ovviamente i piccoli, da soli, non possono farcela. Entrambi gli epiloghi sono condizionati dall'immaturità dei protagonisti. Nel romanzo di Calvino, Pin si allontana con Cugino, il partigiano misogino che pur avendogli appena ucciso la sorella lo protegge ${ }^{10}$. Nel romanzo di Berto, Daniele, abbandonata Carla con la piccola handicappata Maria, sale sul primo treno e in un gesto di ispirazione religiosa dona al mondo i suoi vestiti prima di lasciarsi cadere sulle rotaie.

L'ottimismo di Calvino è solo apparente e allude alla scelta volontaria di un avvenire di difficile crescita e di scoperte dolorose, mentre il pessimismo di Berto pare collegato al rifiuto di crescere, alla tragica resa a un modo infantile di intendere la responsabilità e il bene.

9. Prostituzione a parte, in Guerra in camicia nera Berto allude a rapporti amorosi più o meno intimi con ragazze minorenni secondo la legge dell'epoca. Cfr. p. 50 e p. 160-171.

10. Si veda C. Nocentini, Italo Calvino and the Landscape of Childhood, NUP, Leeds, Maney, 2000, p. 27. 